A C T A C H E M I C A S C A N D I N A V I C A $25 \quad(1971) \quad 470-472$

\title{
Thermodynamic Functions from Spectroscopic Data for Some Alkali Fluorides
}

\author{
B. N. CYVIN, S. J. CYVIN and D. BHOGESWARA RAO \\ Technical University of Norway, N-7034 Trondheim, Norway
}

A. SNELSON

IIT Research Institute, Chicago, Illinois 60616, USA

\begin{abstract}
Thermodynamic functions were calculated from spectroscopic data for ${ }^{7} \mathrm{Li}_{2} \mathrm{~F}_{2}, \mathrm{Na}_{2} \mathrm{~F}_{2}$, and $\mathrm{LiNaF}_{2}$. The results for ${ }^{7} \mathrm{Li}_{2} \mathrm{~F}_{2}$ and $\mathrm{Na}_{2} \mathrm{~F}_{2}$ are comparable with the data of JANAF tables. For the last molecule $\left(\mathrm{LiNaF}_{2}\right)$ the thermodynamic functions have not been given before.
\end{abstract}

$\mathrm{H}$ armonic force field analyses have recently been performed ${ }^{1}$ for $\mathrm{Li}_{2} \mathrm{~F}_{2}$, $\mathrm{Na}_{2} \mathrm{~F}_{2}$, and $\mathrm{LiNaF}_{2}$ on the basis of observed infrared spectra ${ }^{2}$ by the matrix isolation technique. Isotopic molecules with ${ }^{6} \mathrm{Li}$ and ${ }^{7} \mathrm{Li}$ were considered in these investigations. In the present work, the adopted structure parameters ${ }^{1}$ along with the assigned vibrational frequencies ${ }^{1,2}$ are used to calculate thermodynamic functions for ${ }^{7} \mathrm{Li}_{2} \mathrm{~F}_{2}, \mathrm{Na}_{2} \mathrm{~F}_{2}$ and ${ }^{7} \mathrm{LiNaF}_{2}$. Standard methods of calculation are employed.

For ${ }^{7} \mathrm{Li}_{2} \mathrm{~F}_{2}$, the following frequencies ${ }^{1,2}$ (in $\mathrm{cm}^{-1}$ ) were used in the calculations: 641, 553, (551), (524), (342), 287. Parenthesized values are calculated frequencies. The calculated thermodynamic functions at temperatures up to $2000^{\circ} \mathrm{K}$ are shown in Table 1 . The results are comparable with those of the JANAF tables, ${ }^{3}$ and are believed to represent slight improvements. Our value of $S^{\circ}\left(1000^{\circ} \mathrm{K}\right)=84.44$ e.u. agrees perfectly with $84.4 \pm 2$ e.u. from Sidorov and Kolosov, ${ }^{4}$ although they had applied vibrational frequencies from Berkowitz, ${ }^{5}$ which have been disproved by later experiments.

For $\mathrm{Na}_{2} \mathrm{~F}_{2}$, the following frequencies ${ }^{1,2}$ (in $\mathrm{cm}^{-1}$ ) were adopted: 380, 363, (320), (293), (287), < 190. Parenthesized values are calculated frequencies. Successive calculations of thermodynamic functions were performed using 190,170 , and $150 \mathrm{~cm}^{-1}$ as the lowest frequency. The results with $170 \mathrm{~cm}^{-1}$ are given in Table 2. The influence from $\pm 20 \mathrm{~cm}^{-1}$ in the lowest frequency is about \pm 0.1 units on $C_{p}$ and $\left(H^{\circ}-H_{0}^{\circ}\right) / T$, and about \pm 0.2 units on $-\left(F^{\circ}-H_{0}^{\circ}\right) / T$ and $S^{\circ}$. Again the present data are believed to give slight im- 
Table 1. Thermodynamic functions (cal degre日 ${ }^{-1} \mathrm{~mol}^{-1}$ ) for ${ }^{7} \mathrm{Li}_{\mathbf{2}} \mathrm{F}_{\mathbf{2}}$.

\begin{tabular}{rrccc}
\hline$T\left({ }^{\circ} \mathrm{K}\right)$ & $C_{p}{ }^{\circ}$ & $\left(H^{\circ}-H_{0}{ }^{\circ}\right) / T$ & $-\left(F^{\circ}-H_{0^{\circ}}\right) / T$ & $S^{\circ}$ \\
\hline 100 & & & & \\
200 & 9.03 & $\mathbf{8 . 1 8}$ & $\mathbf{4 1 . 2 6}$ & $\mathbf{4 9 . 4 3}$ \\
300 & 12.93 & $\mathbf{9 . 5 7}$ & $\mathbf{4 7 . 3 1}$ & $\mathbf{5 6 . 8 8}$ \\
400 & 15.69 & 11.20 & $\mathbf{5 1 . 5 0}$ & $\mathbf{6 2 . 6 9}$ \\
500 & 17.20 & 12.53 & $\mathbf{5 4 . 9 1}$ & $\mathbf{6 7 . 4 4}$ \\
600 & 18.05 & 13.56 & $\mathbf{5 7 . 8 2}$ & 71.38 \\
700 & 18.56 & 14.35 & $\mathbf{6 0 . 3 7}$ & $\mathbf{7 4 . 7 2}$ \\
800 & 18.89 & 14.98 & 62.63 & $\mathbf{7 7 . 6 0}$ \\
900 & 19.11 & 15.48 & $\mathbf{6 4 . 6 6}$ & $\mathbf{8 0 . 1 4}$ \\
1000 & 19.26 & 15.89 & $\mathbf{6 6 . 5 1}$ & 82.40 \\
1100 & 19.38 & 16.24 & 68.20 & $\mathbf{8 4 . 4 4}$ \\
1200 & 19.46 & 16.53 & 69.76 & $\mathbf{8 6 . 2 9}$ \\
1300 & 19.52 & 16.77 & 71.21 & $\mathbf{8 7 . 9 8}$ \\
1400 & 19.57 & 16.99 & 72.56 & $\mathbf{8 9 . 5 5}$ \\
1500 & 19.62 & 17.17 & $\mathbf{7 3 . 8 3}$ & $\mathbf{9 1 . 0 0}$ \\
1600 & 19.65 & 17.34 & $\mathbf{7 5 . 0 2}$ & $\mathbf{9 2 . 3 6}$ \\
1700 & 19.67 & 17.48 & $\mathbf{7 6 . 1 4}$ & $\mathbf{9 3 . 6 3}$ \\
1800 & 19.70 & 17.61 & $\mathbf{7 7 . 2 1}$ & $\mathbf{9 4 . 8 2}$ \\
1900 & 19.72 & 17.73 & $\mathbf{7 8 . 2 2}$ & $\mathbf{9 5 . 9 4}$ \\
2000 & 19.73 & 17.83 & $\mathbf{7 9 . 1 8}$ & $\mathbf{9 7 . 0 1}$ \\
\hline
\end{tabular}

Table 2. Thermodynamic functions (cal degree ${ }^{-1} \mathrm{~mol}^{-1}$ ) for $\mathrm{Na}_{\mathbf{2}} \mathrm{F}_{\mathbf{2}}$.

\begin{tabular}{rrrrr}
\hline$T\left({ }^{\circ} \mathrm{K}\right)$ & $C_{p}^{\circ}$ & $\left(H^{\circ}-H_{0}^{\circ}\right) / T$ & $-\left(F^{\circ}-H_{0}^{\circ}\right) / T$ & $S^{\circ}$ \\
\hline 100 & 11.3 & 8.9 & 45.3 & \\
200 & 16.1 & 11.4 & 50.8 & 64.5 \\
300 & 17.9 & 13.3 & 54.1 & 70.0 \\
400 & 18.7 & 14.6 & 56.4 & 76.2 \\
500 & 19.1 & 15.5 & 58.1 & 80.4 \\
600 & 19.3 & 16.1 & 59.6 & 83.9 \\
700 & 19.5 & 16.6 & 60.8 & 86.9 \\
800 & 19.6 & 16.9 & 61.9 & 89.5 \\
900 & 19.6 & 17.2 & 62.8 & 91.8 \\
1000 & 19.7 & 17.5 & 63.6 & 93.9 \\
1100 & 19.7 & 17.7 & 64.4 & 95.8 \\
1200 & 19.7 & 17.8 & 65.1 & 97.5 \\
1300 & 19.8 & 18.0 & 65.7 & 99.1 \\
1400 & 19.8 & 18.1 & 66.3 & 100.5 \\
1500 & 19.8 & 18.2 & 66.9 & 101.9 \\
1600 & 19.8 & 18.3 & 67.4 & 103.2 \\
1700 & 19.8 & 18.4 & 67.9 & 104.4 \\
1800 & 19.8 & 18.5 & 68.3 & 105.5 \\
1900 & 19.8 & 18.6 & 68.7 & 106.6 \\
2000 & 19.8 & 18.6 & 69.1 & 107.6 \\
\hline
\end{tabular}

provements of the JANAF tables. ${ }^{3}$ Our value of $S^{\circ}\left(1000^{\circ} \mathrm{K}\right)=93.9 \pm 0.2$ e.u. agrees with 94 e.u. from Sidorov and Kolosov, ${ }^{4}$ who again had applied frequencies from Berkowitz. ${ }^{5}$ 
For ${ }^{7} \mathrm{LiNaF}_{2}$, the following frequencies ${ }^{1,2}$ (in $\mathrm{cm}^{-1}$ ) were used in the calculations: $660,589,376,326,(321), 238$. Thermodynamic functions for this molecule have not been given before. The results of our calculations are shown in Table 3.

Table 3. Thermodynamic functions (cal degree ${ }^{-1} \mathrm{~mol}^{-1}$ ) for ${ }^{2} \mathrm{LiNaF}_{2}$.

\begin{tabular}{|c|c|c|c|c|}
\hline$T\left({ }^{\circ} \mathrm{K}\right)$ & $C_{p}^{\circ}$ & $\left(H^{\circ}-H_{0}^{\circ}\right) / T$ & $-\left(F^{\circ}-H_{0}^{\circ}\right) / T$ & $S^{\circ}$ \\
\hline 100 & 9.90 & 8.41 & 45.00 & 53.41 \\
\hline 200 & 14.14 & 10.29 & 51.39 & 61.68 \\
\hline 300 & 16.50 & 12.01 & 55.90 & 67.91 \\
\hline 400 & 17.74 & 13.30 & 59.54 & $\mathbf{7 2 . 8 4}$ \\
\hline 500 & 18.42 & 14.26 & 62.62 & 76.88 \\
\hline 600 & 18.83 & 14.99 & 65.29 & 80.28 \\
\hline 700 & 19.09 & 15.56 & 67.64 & 83.20 \\
\hline 800 & 19.26 & 16.01 & 69.75 & 85.76 \\
\hline 900 & 19.39 & 16.38 & 71.66 & 88.04 \\
\hline 1000 & 19.48 & 16.69 & $\mathbf{7 3 . 4 0}$ & 90.09 \\
\hline 1100 & 19.54 & 16.94 & $\mathbf{7 5 . 0 0}$ & 91.95 \\
\hline 1200 & 19.60 & 17.16 & 76.49 & 93.65 \\
\hline 1300 & 19.64 & 17.35 & $\mathbf{7 7 . 8 7}$ & 95.22 \\
\hline 1400 & 19.67 & 17.52 & 79.16 & 96.68 \\
\hline 1500 & 19.69 & 17.66 & 80.37 & 98.03 \\
\hline 1600 & 19.71 & 17.79 & 81.52 & 99.31 \\
\hline 1700 & 19.73 & 17.90 & 82.60 & 100.50 \\
\hline 1800 & 19.75 & 18.00 & 83.63 & 101.63 \\
\hline 1900 & 19.76 & 18.10 & 84.60 & 102.70 \\
\hline 2000 & 19.77 & 18.18 & 85.53 & 103.71 \\
\hline
\end{tabular}

\section{REFERENCES}

1. Snelson, A., Cyvin, B. N. and Cyvin, S. J. To be published.

2. Cyvin, B. N., Cyvin, S. J. and Snelson, A. J. Phys. Chem. 74 (1970) 4338.

3. JANAF Thermochemical Tables, Ed., D. R. Stull, Dow Chemical Co., Midland, Mich.

4. Sidorov, L. N. and Kolosov, E. N. Russ. J. Phys. Chem. (English Transl.) 42 (1968) 1382.

5. Berkowitz, J. J. Chem. Phys. 32 (1960) 1519.

Received June 1, 1970. 